\title{
SCREENING OF PATIENTS IN MANAGING PRESBYOPIC AGE GROUP CATARACT PATIENTS AND BENEFITS OF ACRYSOF MULTIFOCAL INTRAOCULAR LENS (DIFFRACTIVE)
}

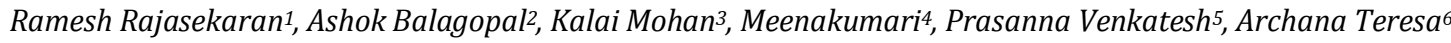

1Chairman, Department of Ophthalmology, Mahathma Eye Hospital, Tiruchirappalli, India.

${ }^{2}$ General Ophthalmologist, Department of Ophthalmology, Mahathma Eye Hospital, Tiruchirappalli, India.

${ }^{3}$ General Ophthalmologist, Department of Ophthalmology, Mahathma Eye Hospital, Tiruchirappalli, India.

${ }_{4}^{4}$ Medical Director, Department of Ophthalmology, Mahathma Eye Hospital, Tiruchirappalli, India.

${ }^{5}$ Fellow-Medical Officer, Department of Ophthalmology, Dr. Sathyan Hospital, Coimbatore, India.

${ }^{6}$ General Ophthalmologist, Department of Ophthalmology, Mahathma Eye Hospital, Tiruchirappalli, India.

\begin{abstract}
BACKGROUND
ABSTRACT

Cataract is the opacity of clear natural lens. Once formed, this has been removed and replaced with monofocal intraocular lens for more than three to four decades. This article focuses on extensive overview of screening of patients for multifocal intraocular lens, indications, relative contradictions and absolute contradictions for multifocal intraocular lens implantation. The methodology of detailed history taking, systematic clinical work-up using appropriate evaluation tools, has been discussed. The outcome of this screening method would considerably increase the number of happy patients with spectacle independence. This article would serve as the best guiding tool for practicing ophthalmic physicians or surgeons to practice multifocal intraocular lens in cataract patients in presbyopic age group. As AcrySof diffractive multifocal intraocular lens design is known to decrease the photic phenomenon and satisfy the requirement of the patient for spectacle independence, its features have also been analysed for the benefit of ophthalmic practitioners.
\end{abstract}

\section{KEY WORDS}

Presbyopic Age Group, AcrySof Diffractive Multifocal Intraocular Lens, Cataract Patients, Photic Phenomenon, Spectacle Independence.

HOW TO CITE THIS ARTICLE: Rajasekaran R, Balagopal A, Mohan K, et al. Screening of patients in managing presbyopic age group cataract patients and benefits of AcrySof multifocal intraocular lens (diffractive). J. Evolution Med. Dent. Sci. 2018;7(46):50495054, DOI: $10.14260 /$ jemds/2018/1123

\section{BACKGROUND}

Cataract is the opacity of clear natural lens. It represents the major cause of inevitable blindness in the world. From 1990 to 2010 the number of blind and visually diminished people due to cataract decreased; however, inspite of increase in the total number of cataract surgeries being performed, the number of untreated cataract patients increased in all the regions especially in Asia. Nowadays, patients demand are becoming more and more challenging. They expect outstanding visual results and perfect vision without spectacles namely both distance and proximity. The 2016 European Society of Cataract and Refractive Surgery Clinical Survey revealed that $43 \%$ of cataract procedures are targeted for monovision. ${ }^{1}$ The 'ideal' intraocular lens (IOL) should restore the patients' vision without difficulties or visual negotiations at all distances. ${ }^{2}$ Cataract surgery and its outcome has improved over the past few decades. Improvisation in surgical techniques, acquisition of instruments with advanced technology, knowledge about choice of IOL formulas and varied options of IOLs available nowadays has made the visual outcome predictable. The expectation of cataract patients for spectacle independence, has lead on to evolution of different types of IOLs.

'Financial or Other Competing Interest': None.

Submission 23-10-2018, Peer Review 03-11-2018,

Acceptance 06-11-2018, Published 12-11-2018.

Corresponding Author:

Dr. Ramesh Rajasekaran,

Chairman,

Mahathma Eye Hospital,

No. 6, Seshapuram, Tiruchirappalli, Tamilnadu, India.

E-mail: meh.drramesh@gmail.com

DOI: $10.14260 /$ jemds/2018/1123

\section{(c) $(1)$}

Optical quality of any multifocal intraocular lens has a compromise E.g. Glare, haloes, other photic phenomenon, which is a trade-off for spectacle independence. Cataract surgery is no more removal of the opacified lens, but it is considered as a refractive procedure which is aimed on good near, intermediate and distant vision. ${ }^{3}$

The purpose of this review is to give an overview of the best practice methodology and the criteria to screen the cataract patients for multifocal intraocular lens. It also focuses on the benefits of AcrySof diffractive IOL which has adopted apodisation technology. This review is only for cataract patients in the presbyopic age group and not for Refractive Lens Exchange.

\section{MATERIALS AND METHODS}

Bibliography research was performed in PubMed and in Medline database published in the past twenty years. More than 100 articles were reviewed and the most recently updated papers were evaluated. The outcome parameters for screening of the cataract patients for suitability of multifocal intraocular lens evolved based on few aspects, such as, History taking, evaluating patient's personality profile, clinical and diagnostic evaluation and also it focuses on benefits of AcrySof diffractive IOL which has adopted apodisation technology.

Preoperative Patient Selection Criteria/Screening of Patients for Suitability of Multifocal Intraocular Lens based on few aspects, such as,-

1. History taking.

2. Evaluating patient's personality profile.

3. Clinical Evaluation.

4. Diagnostic Evaluation. 


\section{History Taking}

History taking includes cautiously and elaborately to understand the patients' personality profile, occupations, hobby, habits, patients expectations, financial status, etc., Multi focal IOLs, though they reduce spectacle dependency, they also lead onto a reduction in image contrast, few perceive photic phenomenon as glare, halos, star burst, etc., as a trade-off for spectacle independence more over Corneal aberrations such as Coma $>0.32$ is avoided ${ }^{4}$. Coma $>0.32$ microns causes intolerable dysphotopsia. Spherical aberrations also cause decreased visual quality. Visual quality of diffractive IOLs is better, when the spherical equivalent is close to plano and post-op regular corneal astigmatism is $0.5 \mathrm{D}$ or less.

\section{a. Hobbies}

Patients with hobbies like cooking, knitting bags, tailoring, swimming, walking, playing video games, playing cricket or golf, knitting sweaters, painting, gardening, watching TV, on social media, aari work and embroidery work all are suitable candidates. Tailors may need to increase their ambient lighting to improve the brightness and contrast sensitivity.

Reading can be categorized as less reading or moderate reading or extensive reading. Candidates with extensive reading especially fine letters e.g. Bible are not ideal candidates. Ideal candidates are those with less or moderate reading which involves not very minute letters. History taking must involve the type of reading material also.

\section{b. Driving}

In History taking, driving is a another important criteria to select the cataract patients for Multi focal IOL. A non-driver is an ideal patient. A driver - if drives in the day time could be ideal for Multi focal IOL implantation. A night time driver- as a hobby or as a profession is to be discouraged for Multi focal IOL. Two or four wheelers driving which involves minimal duration or mileage even in the night time in a friendly point and not an absolute contraindication.

\section{c. Spectacle History}

Spectacle history should be documented and their prior prescription should be analysed. Low myopes are not ideal patients as they have enjoyed excellent near vision with spectacle independence.. It's difficult to match their near vision which they enjoyed with low myopia. The spectacle power must be noted and documented. All the previous prescriptions must be analysed and documented. More the precision in history taking, more perfection in pre-op assessment, better is the visual outcome.

\section{d. Past History}

Any past history of eye ailments ex. Retinal disorders, lasers for retinal diseases, retinal surgeries must be elicited.

\section{e. Medical History}

Medical history like diabetic history must be elicited because advanced diabetic retinopathy could be an absolute contra indication.

\section{f. Astigmatism and Multi focal IOL Selection Criteria} Corneal astigmatism of $>1.25 \mathrm{D}$ is prevalent is approximately $30 \%$ of eyes with cataract, $16-18$ patients with regular astigmatism.5,6,7
An ideal candidate is one who only has spherical error. But patients with refractive error of $<0.75 \mathrm{D}$ cylinder do well with multifocal IOLs. Patients with more than one diopter cylinder, image quality degrades. Pre-operative objective and subjective refraction, power of already used prescribed glasses, pre-op patient's prescriptions should be tabulated and assessed.

\section{Evaluating Patient's Personality Profile}

Pre-op counselling, understanding patient's personality profile, visual requirement in their day to day life, expectation of individual patients etc is essential., Monofocal patients never face situations of optical aberrations, need for enhancement etc. Multifocal IOL patients, have to be extensively counselled in this regards pre-operatively.

Patients have to be characterized as ideal and non-ideal patients during the chair time. Ideal patient is a causal natured person, easy going personality accepting small visual compromises, willing to accept the unexpected post-op surprises which is not vision threatening. Patients who is keen on a very cordial "patient - doctor" relationship, moderate to high hypermetropes, patients with less of intermediate jobs and more of near job are ideal. Higher the age group better is the acceptance of Multifocal IOL and its visual outcome. Patients retried from their job and are involved in less near vision job, but has more other hobbies apart from reading are ideal individuals for easily adapting to Multi focal technology. Patients with Tremors due to parkinsonism, dementia, forget fullness for spectacles are ideal patients. Patients with dementia- informed consent is necessary. In elderly senior citizen still employed in Near vision task, make sure his Near vision task occupies less of his day hours. "Always finding fault" syndrome patients, patients who is concerned with sharp, clear vision are ideal only for monofocals. Night time drivers as an occupation or as a hobby are not an ideal candidate. Multi focal IOL is pupil dependent. Optical aberrations come into play when the pupil dilates during night time.

\section{Clinical Evaluation}

Good visual acuity assessment of both the eyes, slit lamp examination, good refraction, a study on previous glass prescriptions, assessing the pupil status, understanding iris configurations and grading of cataract must be done. Any pupillary abnormalities (posterior synechia, mid dilated pupil status, sphincter atrophy, PXF, etc) should be documented for decision making at a later stage. Good fundus evaluation should be done whenever possible. Attempts to examine the periphery $\mathrm{pf}$ the retina with indirect ophthalmoscopy is essential. If any retinal hole, tear, breaks noted-this must be lasered prior to cataract surgery. In mature cataracts B Scan and visual acuity assessment using RAM \{Retina Acuity Meter\} needs to be carried out.

\section{Diagnostic Evaluation}

\section{a. Dry Eyes and Multifocal IOL}

Identifying mild to moderate dry eye, meibomian gland dysfunction, is essential. If not identified and treated preoperatively, may result in poor quality of vision, in spite of excellent surgical outcome. Extensive dry eye evaluation, Meibomian gland disfunction identification should be done 
with a structured dry eye workup sheet to avoid post-op unhappy patients. Patients with mild to moderate dry eye underline cause should be identified and treated prior to multifocal IOL implantation. Severe dry eye is a strong contra indication. Pre-operative IOL formula work up should be done and the appropriate formula chosen only after successfully managing the patient.

\section{b. Corneal Considerations and MF IOL}

Corneal dystrophies, central corneal scars, fleshy and progressive pterygium extending into the cornea or cases where irregular astigmatism has been induced by a pterygium, or a pterygium excised scar is an absolute contra indication. Peripheral asymptomatic scars with no induced astigmatism can be considered. Conditions like Fuch's Endothelial Dystrophy leads on to decreased contrast sensitivity-better avoided in patient selection criteria8. Hence role of Specular microscope and careful interpretation of the endothelial cell number and morphology as a pre-op assessment tool is emphasized.

\section{c. Macular Function}

Visual assessment in patient is a static phenomenon. But, macular function test with Pin Hole test, super pinhole test, Multiple Pinhole test, Retinal Acuity Metre, OCT macula test are good assessment tools ${ }^{2}$. Success of Multifocal IOL depends on excellent reading speed. This is a dynamic process rather than static Visual Acuity assessment. Any abnormality of the fovea and macula is a strong contra-indication for Multi focal IOL.

\section{d. Biometry}

Immersion Biometry or optical biometry are good tools to evaluate the IOL formulas. In situations where Optical Biometry is not available or accessible; immersion biometry is a good choice but certainly not the applanation Biometry.

Optical biometers are more precise and less technician dependent than ultrasound measurement.9,10\&11

SRK-T formulas are good for normal sized and long eyes. ${ }^{12 \& 13}$ Hoffer $Q$ and Haigis for small and very small eyes. Holladay-2 and Hairgis are good for extreme axial lengths. Modern formulas like Barret Universal-2 formula or Olson formula does not have a wide spread use and report still awaited. Validation guideline should be understood and applied carefully whenever the IOL power elicited.

\section{e. Retinal Diseases and Multi Focal IOL}

Any known H/o of macular or retinal pathologies, ARMD, ERMS, Retinitis pigmentosa etc., are strong contraindications. ${ }^{44,15,16,17 \& 18}$ All the retinal pathologies decrease the contrast which brings down the performance of Multifocal IOL considerably. Patients who have undergone any retinal surgical procedures is a contra indication (Absolute).

Significant Diabetic retinopathy, documentation of rapidly progressing Diabetic retinopathy, any form of Diabetic. Maculopathy, maculopathy received focal or Grid laser Photocoagulation, Proliferative diabetic retinopathy who has received Panretinal photocoagulation are some absolute contra-indications for Multi focal IOL implantations. Diabetic patient with good sustained control of diabetes with no diabetic retinopathy or mild diabetic retinopathy is not a contra-indication. Diabetic patient which poor metabolic control, or rapidly progressive diabetic retinopathy is a relative contraindication. Diabetic patients with NO DR, mild or moderate DR or patients where there is no co-existing systemic comorbidities like diabetic nephropathy or cardiopathy could be taken into consideration for Multi focal IOL. Any other documented or known H/o retinal or macular pathologies are strong contra-indications for MF IOL. Denser cataracts where OCT macula\& RAM is not possible makes difficult in decision making and the decision more towards monofocal IOL is encouraged.

\section{f. Optic Nerve Pathologies and Multi Focal IOL}

Visual acuity, contrast sensitivity, colour perception, visual field - is all reduced in patients with optic nerve pathology19\&20. Optic nerve pathology can be evaluated by Visual acuity charts, pupil by direct and consensual Reflex, Colour vision testing, Visual field analysis by automated perimeter and OCT optic nerve head evaluation. If any abnormality of optic nerve is detected, then Multi focal IOL selection is a contra-indication. Visual activity, contrast sensitivity, colour perception, visual fields, is all reduced in patients with optic nerve pathology.

\section{g. Surgical Techniques - A Tribute to Multi Focal IOL}

A limbal corneal incision with minimal vascularity, helps in rapid healing compared to clear corneal or limbal. A temporal incision is perfect unless the steep meridian is superiorly located. $2.2 \mathrm{~mm}$ incision and a square incision with a guard is used. A good architecture of the would contributes to decreasing the pre-existing minimal astigmatism of $<-0.5 \mathrm{D}$ to $0.75 \mathrm{~d}$ ). Using a rhexis marker of $5.5 \mathrm{~mm}$ well centred on the cornea, helps to perform a well centered, circular, continuous, curvilinear, rhexis of $5 \mathrm{~mm}$ which overlaps $1 \mathrm{~mm}$ all around a $6 \mathrm{~mm}$ optic. Proper centration of Multi focal iol and effective lens position cannot be under estimated. Asymmetrical rhexis, especially in mature cataracts and hyper mature cataracts, deformed haptics and zonular weakness causes IOL decentration ${ }^{21}$. Zonular weakness and zonular dialysis in PXF patients, hard cataract, etc is another challenge while implanting Multi focal IOL. CTR can be used to distribute forces around the equator to stabilize the IOL. CTR can be used only when there is no anterior or posterior - capsule tears and only when the capsular bag is intact. 22 If there is an accidental opening of posterior capsule a successful Posterior- continuous- curvilinear circular capsulorhexis enhances the possibility of implanting the MFIOL in the bag. When there is an anterior capsular tear the IOL can be placed only if it is a single radial tear, no extension into posterior capsule still an experienced surgeon can place it positioning the haptic perpendicular to the tear ${ }^{23}$. It may or may not lead to IOL decentration in the long run.24,25\&26 Converting to posterior continuous, curvilinear, capsulorhexis placement provides a continuous and strong margin for Multi focal IOL placement. If there is a large PCR or PCR with considerable vitreous loss, even after successful vitrectomy, Multi focal IOL placement is going to produce an unhappy patient. Implanting single piece Multi focal IOL in the sulcus in a panic attempt, to be avoided as this will lead on to recurrent post op inflammation, pigment dispersion and haemorrhage. Hence on this occasion monofocal IOL is advocated. Ideal phaco-dynamics to avoid post-op corneal oedema or striate 
keratopathy is advocated so that visual rehabilitation is faster and matches the patients expectation. Abundant low molecular weight dispersive visco-elastic and soft shell techniques to be followed to minimize endothelial cell loss in this premium surgery.

\section{h. Keratometry:}

Manual keratometry, automated keratometry, partial coherence interferometry, keratometry and topography was performed. Topography gives information about regularity of the astigmatism. Later it might help us in laser vision correction enhancements. Patients with irregular astigmatism are not ideal candidates. Limbal relaxing incision, opposite clear corneal incision are not permanent remedies and not recommended in Multi focal-IOLs.

\section{i. Pre-Op OCT}

OCT is more reliable than indirect ophthalmoscopy, direct ophthalmoscopy, slit lamp examination with 90D lens as a pre-op evaluation of macula for any type of maculopathy as a tool in predicting tool in predicting outcome as well as in patient selection. OCT is real time, high resolution. It helps to eliminate several patients for multi focal IOLs is in decreasing the number of unhappy patients. Certainly patients taken up for Multi focal IOL without OCL pre - Op- there could be large turnover of unhappy patients due to retinal pathology and leading on to IOL explanation.

A happy and satisfied Multi focal IOL patients reflects the following- there was no per-op complication, IOL power calculation was perfect, appropriate formula was used, appropriate incision which takes care of minimal pre-existing astigmatism and Patient selection criteria has been deeply looked into.

\section{j. Zonular Weakness}

Tilt of the IOL affects the distribution of light between the distant and near foci. Thus visual outcome is compromised. In non-rotational symmetric IOL, the impact of decentration is more compromised in refractive - diffractive IOLs'.

\section{k. Angle Kappa}

It is the angle between the papillary axis and the visual axis. A large angle kappa gives glare or haloes. Photic phenomenon is heavily expressed in temporal decentration of IOL. Angle kappa $>5^{\circ}$ is a contra - indication. Angle kappa can be measured by synoptophore and optical biometry.

\section{Pupil Size and Multi Focal IOL}

Clinical evaluation of pupil should be studied and documented under scotopic, mesopic and photopic conditions. In all the patients- document rigid, miotic pupil, PXF pupil. Irregular pupil, post-traumatic mydriatic pupil, irregular pupil due to complicated cataract, eccentric pupils and key shaped pupil (iris coloboma) atrophy iris due to various reasons are absolute contradictions. This is pupil dependent IOL Patient with pupil irregularity are prone for increased glare and other photic phenomenon. Rhexis problems and associated zonular weakness resulting in decentred IOL lead to alarming outcome in the post-op. Intraocular stray lights increases with increasing pupil diameter. Using iris hooks in small pupils and inducing iatrogenic Mydriasis leads to alarming reflections and optical aberrations.

\section{m. Unhappy Patients with Multi Focal}

When patients do not achieve visual goals, they are unhappy patients. Less or no photic phenomenon, adjustable and accommodative personality characteristics of the patient, visual outcome matching the patients' expectations of spectacle independence contributes to patients satisfaction. Spectacle independence is one of the major demands of the patients.

\section{MF IOL and Monocularity}

In patients who has monofocal IOL in one eye, if he/she is unassuming with minimal or no over expectation, he could be opted for Multi focal IOL. These patients should be educated to expect bilateral multifocality. When all the credential are analysed into every patients the incidence of unhappy patients will be minimized considerably.

\section{Apodized Diffractive Central Zone and Refractive Periphery Multifocal Lens}

Multifocal's working principle is based on the creation of two focal points by using diffractive orders - order 0 and order 1 . The power corresponding to the order 0 is used to image distant objects whereas the order 1 is used for near vision. Reasons for side effects like glare and haloes are due to image formed by one focal on the retina and it will have a de-focused image from the other focus superimposed. This results in the optical image blur. Due to lens design of diffractive IOLs, patients have lower contrast sensitivity mainly due to forward dispersion and higher order aberration. Apodisation of central diffractive zone by gradually reducing the number of diffractive steps in width and height creates a smooth transition between far, intermediate and near focal points ${ }^{27}$.

Percentage of light reaching the near focus and distant focus depending on patient's pupil size can be modified in this apodized type of technology. So in right light, when pupil is small the IOL distributes the light energy equally between distance and near far sight, whereas in dim light situation, when the pupillary size increases less light is directed to near focus and more for distant focus. Apodized technology, considering the outcome analysis, can be discussed in various heads. Such as-

i. Patient's satisfaction - in this, spectacle independence is very high in apodized IOL, for distance, intermediate and near compared to other technology,

ii. Vision improvement - how is the visual improvement present in all types of lighting condition, pupillary size is the hallmark for visual improvement,

iii. Contrast sensitivity - is a most vital thing. Changes in the light levels can reduce contrast sensitivity, mainly at higher frequencies. This lens has normal contrast sensitivity range between photopic and it has reduced contrast sensitivity range in mesopic conditions. The depth of the focus is better with apodized IOL but if the astigmatism is more than point side diopter, depth of focus decreases. The proper preoperative analysis should never induce any surgically induced astigmatism. 
The more important thing about the visual improvement is not just the visual acuity improvement alone. It's again the reading speed that decides the lens performance. Because visual assessment is not concerned as a good indicator of patient's visual clarity and quality. Because it is measured in static conditions, whereas reading speed indicates the dynamic property of apodized lens and its management in presbyopic correction post-surgery. The unwanted visual symptoms like glare and hallows which reduces the visual quality in minimized with diffractive center and refractive periphery apodized IOL ReSTOR lenses. These conditions increase when the pupillary diameter becomes larger especially in night conditions.

The lens material allow maximum light into retina and they decrease forward scattering and eliminates the internal reflections also resulting in better optical clarity and quality. Intraocular light scattering increases with pupillary diameter mainly and if surgically the rhexis is smaller. Many times, a surgeon has a tendency for a small rhexis when the multifocal lens especially at the beginning stage of the surgery. Preoperatively, anterior corneal aberration profile to assess and avoid conditions like coma and astigmatism are all perfectly screened. But IOLs with spherical profile can increase spherical aberrations. Hence in order to reduce this higher order aberrations, aspheric profiles have been designed and they have been proved to be very effective. But then, we should have it in mind that asphericity is lost when we operate patients with small rigid pupils. This should be considered and in preoperative assessment pupillary size has to be properly assessed. This gradual decrease in the apodisation of multifocal IOLs from center to the periphery in width and height allows a decrease in ocular operations, hence maximizing the lens performance. In laboratories which a surgeon may not get access to these scenarios, point spread function (PSF) has been studied. This study has revealed that in eyes with high power apodized diffractive multifocal IOLs, the optical quality comes down. So this we need ti have in mind when operate on patients with refractive errors.

The next technology is modulation transfer function (MTF) which describe how all facial frequencies are transmitted by an optical system. To conclude that, the present generation apodized diffractive multifocal IOL provides a comprehensive visual improvement foe distance, intermediate and near. The gradual transition of the diffractive steps from the center to the periphery avoids variation in contrast sensitivity and produces fewer optical aberrations minimizing glare and hallows, especially in night condition when the pupillary size increases.

\section{CONCLUSION}

Multi focal IOL has undergone various transformation, from refractive to diffractive apodisation and from +4 add to +3 add and +2.5 add. All this evolution aiming on spectacle independence for near, intermediate and distance vision. Understanding about pre-operative evaluation of the patients extensively, has helped to reduce the post-operative unhappy patients.

Hence the understanding of the Multi focal IOL technology and behaviour is essential for every surgeon prior to planning Multi Focal IOL implantation. IOL selection aiming at reduction in photic phenomenon has helped successful outcome in apodisation technology. Extreme and excellent work up during patient selection, and stringently following guidelines for absolute contra indications adds on to the number of happy patients after multi focal IOL implantations.

\section{REFERENCES}

[1] European Society of Cataract and Refractive Surgery Clinical Survey. http://www.eurotimes.org/escrsclinical-survey-2016-results/accessed 14 December 2017.

[2] Braga-Mele R, Chang D, Dewey S, et al. Multifocal intraocular lenses: relative indication and contraindications for implantation. J Cataract Refract Surg 2014;40(2):313-22.

[3] Zvornicanin J, Zvornicanin E. Premium intraocular lenses: the past, present and future. The J of Current Ophthalmology 2018: p. 1-10.

[4] Visser N, Nuijts RM, de Vries NE, et al. Visual outcomes and patient satisfaction after cataract surgery with toric multifocal intraocular lens implantation. J Cataract Refract Surg 2011;37(11):2034-42.

[5] Ferrer-Blasco T, Montes-Mico R, Peixoto-de-Matos SC, et al. Prevalence of corneal astigmatism before cataract surgery. J Cataract Refract Surg 2009;35(1):70-5.

[6] Hoffer KJ. Biometry of 7, 500 cataractous eyes. Am J Ophthalmol 1980;90(3):360-8.

[7] Hoffmann PC, Hutz WW. Analysis of biometry and prevalence data for corneal astigmatism in 23, 239 eyes. J Cataract Refract Surg 2010;36(9):1479-85.

[8] Woodward MA, Randleman JB, Stulting RD. Dissatisfaction after multifocal intraocular lens implantation. J Cataract Refract Surg 2009;35(6):9927.

[9] Findl O, Kriechbaum K, Sacu S, et al. Influence of operator experience on the performance of ultrasound biometry compared to optical biometry before cataract surgery. J Cataract Refract Surg 2003;29(10):1950-5.

[10] Moeini H, Eslami F, Rismanchian A, et al. Comparison of ultrasound and optic biometry with respect to eye refractive errors after phacoemulsification. JRMS 2008;13(2):43-7.

http://journals.mui.ac.ir/jrms/article/

view/1042/719. Accessed October 6, 2013.

[11] Rajan MS, Keilhorn I, Bell JA. Partial coherence laser interferometry vs conventional ultrasound biometry in intraocular lens power calculations. Eye (Lond) 2002;16(5):552-6.

http:// www.nature.com/eye/journal/v16/n5/pdf/6700157 a.pdf. Accessed October 6, 2013.

[12] Ghanem AA, El-Sayed HM. Accuracy of intraocular lens power calculation in high myopia. Oman J Ophthalmol 2010;3(3):126-30.

http://www.ojoonline.org/temp/OmanjOphthalmol3 3126-35191_094631.pdf. Accessed October 6, 2013

[13] Donoso R, Mura JJ, Lopez M, et al. Buscando la emetropia en cirugia de catarata con la formula mas indicada para cada ojo segun su longitud axial [Looking for the best IOL power calculation formula according to the eye length]. Arch Soc Esp Oftalmol 2003;78:477-80. http://scielo.isciii.es/scielo.php? 
scriptZsci_arttext\&pidZS0365-

66912003000900004\&lngZen \&nrmZiso. Accessed October 6, 2013.

[14] Friedman DS, O'Colmain BJ, Muñoz B, et al. Prevalence of age-related macular degeneration in the United States. Arch Ophthalmol 2004;122(4):564-72. http://archopht.

jamanetwork.com/data/Journals/OPHTH/9922/EEB3 0090. pdf. Accessed October 6, 2013.

[15] Kleiner RC, Enger C, Alexander MF, et al. Contrast sensitivity in age-related macular degeneration. Arch Ophthalmol 1988;106(1):55-7.

[16] Stangos N, Voutas S, Topouzis F, et al. Contrast sensitivity evaluation in eyes predisposed to agerelated macular degeneration and presenting normal visual acuity. Ophthalmologica 1995;209(4):194-8.

[17] Chew EY, Sperduto RD, Milton RC, et al. Risk of advanced age-related macular degeneration after cataract surgery in the Age-Related Eye Disease Study: AREDS report 25. Ophthalmology 2009;116(2):297303.

[18] Fraser-Bell S, Guzowski M, Rochtchina E, et al. Fiveyear cumulative incidence and progression of epiretinal membranes: the Blue Mountains Eye Study. Ophthalmology 2003;110(1):34-40.

[19] Duke-Elder S, Scott GI. Neuro-Ophthalmology. In: Duke Elder DS, edr. System of Ophthalmology. Vol. 12. London, UK: Kimpton 1971: p. 11-5.

[20] Miller NR. Walsh and Hoyt's Clinical neuroophthalmology. Vol. 1. $4^{\text {th }}$ edn. Baltimore: Williams \& Wilkins 1985: p. 212-25, 329-42.
[21] Walkow T, Anders N, Pham DT, et al. Causes of severe decentration and subluxation of intraocular lenses. Graefes Arch Clin Exp Ophthalmol 1998;236(1):9-12.

[22] Alio JL, Elkady B, Ortiz D, et al. Microincision multifocal intraocular lens with and without a capsular tension ring: optical quality and clinical outcomes. J Cataract Refract Surg 2008;34(9):146875.

[23] Alio JL, Plaza-Puche AB, Pinero DP. Rotationally asymmetric multifocal IOL implantation with and without capsular tension ring: refractive and visual outcomes and intraocular optical performance. J Refract Surg 2012;28(4):253-8.

[24] Davison JA. Analysis of capsular bag defects and intraocular lens positions for consistent centration. J Cataract Refract Surg 1986;12(2):124-9.

[25] Oner FH, Durak I, Soylev M, et al. Long-term results of various anterior capsulotomies and radial tears on intraocular lens centration. Ophthalmic Surg Lasers 2001;32(2):118-23.

[26] Haigh PM, Lloyd IC, Lavin MJ. Implantation of foldable intraocular lenses in the presence of anterior capsular tears. Eye (Lond) 1995;9(Pt 4):442-5. http://www.nature.com/eye/journal/ v9/n4/pdf/eye1995103a.pdf. Accessed October 6, 2013.

[27] Tomas-Juan J. Multifocal IOLs with apodized diffractive central zone and refractive periphery: optical performance and clinical outcomes. J Emmetropia 2014;5:155-66. 